\title{
A DEGREE THEORY FOR LOCALLY COMPACT PERTURBATIONS OF FREDHOLM MAPS IN BANACH SPACES
}

\author{
PIERLUIGI BENEVIERI AND MASSIMO FURI
}

Received 16 December 2003; Accepted 21 January 2005

We present an integer valued degree theory for locally compact perturbations of Fredholm maps of index zero between (open sets in) Banach spaces (quasi-Fredholm maps, for short). The construction is based on the Brouwer degree theory and on the notion of orientation for nonlinear Fredholm maps given by the authors in some previous papers. The theory includes in a natural way the celebrated Leray-Schauder degree.

Copyright (c) 2006 P. Benevieri and M. Furi. This is an open access article distributed under the Creative Commons Attribution License, which permits unrestricted use, distribution, and reproduction in any medium, provided the original work is properly cited.

\section{Introduction}

In [1] we gave a new definition of oriented degree for (nonlinear) Fredholm maps of index zero between real Banach manifolds. Our approach, based on the simple algebraic idea of giving an orientation to Fredholm linear operators of index zero, extends and simplifies the well known Elworthy-Tromba construction (see $[5,6]$ ). Analogously to the degree theory developed by Fitzpatrick, Pejsachowicz and Rabier in $[7,8]$, in our method we introduce a concept of orientation for Fredholm maps of index zero, avoiding, in this way, the use of any Fredholm structure and any related concept of infinite-dimensional orientation on manifolds; notions that are needed in the Elworthy-Tromba theory. (A comparison between our approach and that of Fitzpatrick, Pejsachowicz and Rabier can be found in $[1,2]$.)

Our degree in [1], which is merely based on the Brouwer degree for maps between finite-dimensional differentiable manifolds, extends the celebrated Leray-Schauder degree theory in the $C^{1}$ case (Fredholm maps are $C^{1}$, by assumption). In order to give a full extension of the Leray-Schauder construction, in this paper we develop a degree theory for locally compact perturbations of Fredholm maps of index zero between (open sets in) Banach spaces (quasi-Fredholm maps, for short). For this purpose we will extend to the quasi-Fredholm maps the notion of orientation introduced in [1] for Fredholm maps of index zero. 
As in [1], our construction will be mainly based on the existing finite-dimensional degree theory (as can be found, e.g., in [16]).

We point out that a degree theory for locally compact perturbations of Fredholm maps has already been developed by Zvyagin and Ratiner in [18]. However, our approach differs from that in [18] since it is not based on the Elworthy-Tromba theory.

\section{Preliminaries}

These preliminaries are devoted to a review of those properties of the Brouwer degree that will be useful for the construction of our degree.

The version of the Brouwer degree we consider here is a slight extension of that exposed by Nirenberg in [16]. In his approach, the degree is an integer assigned to any triple $(f, U, y)$, where $f, U$ and $y$ are as follows. Given two oriented $C^{\infty}$ real manifolds $M$ and $N$ of the same (finite) dimension, $U$ is an open subset of $M, f: M \rightarrow N$ is a continuous map that is proper on the closure $\bar{U}$ of $U$, and $y$ is an element of $N$ such that $y \notin f(\partial U)$. We point out that Nirenberg's approach is still valid if the manifolds $M$ and $N$ are supposed to be $C^{1}$, which is the case we consider in this paper.

We find worthwhile to stress that the construction of Nirenberg includes the two classical approaches to the finite-dimensional degree: one regarding maps defined on the closure of bounded open subsets of $\mathbb{R}^{n}$ and the other concerning maps between compact manifolds (for extensive expositions of the Brouwer degree theory we refer to $[4,10,13,14,16])$.

The assumptions required by Nirenberg can be easily weakened. In fact, given two oriented $C^{1}$ real manifolds $M$ and $N$ of the same dimension and a continuous map $f$ : $M \rightarrow N$, the degree can be defined for any triple $(f, U, y)$ with $U$ open in $M$ and $f^{-1}(y) \cap$ $U$ compact. More precisely, given an open subset $W$ of $U$ such that $\left(f^{-1}(y) \cap U\right) \subseteq W$ and $\bar{W} \subseteq U$, if $\left.f\right|_{\bar{W}}$ is proper, the degree of $(f, U, y)$ is defined as

$$
\operatorname{deg}(f, U, y)=\operatorname{deg}(f, W, y) .
$$

The excision property implies that $\operatorname{deg}(f, U, y)$ is well-defined, in the sense that the righthand side of the above equality does not depend on $W$.

The classical properties of the Brouwer degree still hold in this extended version. The proof can be easily obtained by a straightforward generalization of the same properties given in [16].

To help the reader we recall here the excision and the homotopy invariance properties, since they will be explicitly used for our construction.

Lemma 2.1 (excision). Let $M$ and $N$ be two oriented $C^{1}$ real manifolds of the same finite dimension and let $f: M \rightarrow N$ be continuous. Consider an element $y \in N$ and an open subset $U$ of $M$ such that $f^{-1}(y) \cap U$ is compact. If $V$ is an open subset of $U$ containing $f^{-1}(y) \cap U$, then

$$
\operatorname{deg}(f, V, y)=\operatorname{deg}(f, U, y)
$$


Lemma 2.2 (homotopy invariance). Let $H: M \times[0,1] \rightarrow N$ be a continuous homotopy. Consider an element $y \in N$ and an open subset $U$ of $M$ such that $H^{-1}(y) \cap(U \times[0,1])$ is compact. Then $\operatorname{deg}(H(\cdot, \lambda), U, y)$ is independent of $\lambda \in[0,1]$.

The above classical version of the homotopy invariance property can be generalized as in Lemma 2.3 below. We obtain a particular extension of Lemma 2.2 that will be used in Section 5.

Lemma 2.3 (extended homotopy invariance). Let $E$ be a real Banach space and $Z$ an oriented $(n+1)$-dimensional submanifold of $E \times[0,1]$ with boundary. Assume that the boundary of $Z$ is

$$
\left(Z_{0} \times\{0\}\right) \cup\left(Z_{1} \times\{1\}\right)
$$

where $Z_{0}$ and $Z_{1}$ are (boundaryless $n$-dimensional) oriented manifolds. Suppose also that the orientation of $Z$ at any point $(x, i), i=0,1$, is the product of the orientation of $Z_{i}$ at $x$ and the canonical orientation of $\mathbb{R}$. Let $N$ be an oriented $n$-dimensional manifold and let $H: Z \rightarrow N$ be continuous. If $y \in N$ is such that $H^{-1}(y)$ is compact, then

$$
\operatorname{deg}\left(H(\cdot, 0), Z_{0}, y\right)=\operatorname{deg}\left(H(\cdot, 1), Z_{1}, y\right)
$$

This version of the homotopy invariance property is not standard since the domain of $H$ is not a product manifold. Nevertheless, the proof can be given as in its classical version.

Another property of the Brouwer degree that we will need is a reduction property (Proposition 2.5 below). Let us recall first some facts regarding the notions of orientation and transversality. Consider a (real) manifold $M$, a (real) vector space $F$ of the same finite dimension and a $C^{1}$ map $g: M \rightarrow F$. Let $F_{1}$ be a subspace of $F$, transverse to $g$. Thus $M_{1}=g^{-1}\left(F_{1}\right)$ is a submanifold of $M$ of the same dimension as $F_{1}$. Assume now that $M$ and $F$ are oriented. One can prove that any orientation of $F_{1}$ induces an orientation on $M_{1}$. Let us sketch how this can be done. Suppose $F_{1}$ oriented and let $x \in M_{1}$ be given. By the transversality assumption, the tangent space to $M_{1}$ at $x$, denoted by $T_{x} M_{1}$, coincides with $\left(g^{\prime}(x)\right)^{-1}\left(F_{1}\right)$. Let $E_{0}$ be any direct complement of $T_{x} M_{1}$ in $T_{x} M$ and let $F_{0}=g^{\prime}(x)\left(E_{0}\right)$. Observe that $g^{\prime}(x)$ maps isomorphically $E_{0}$ onto $F_{0}$ and that $F=F_{0} \oplus F_{1}$. Let $F_{0}$ be endowed with the orientation such that a positively oriented basis of $F_{0}$ and a positively oriented basis of $F_{1}$, in this order, form a positively oriented basis of $F$. Then, orient $E_{0}$ in such a way that $\left.g^{\prime}(x)\right|_{E_{0}}: E_{0} \rightarrow F_{0}$ is orientation preserving. Finally, orient $T_{x} M_{1}$ in such a way that a positively oriented basis of $E_{0}$ and a positively oriented basis of $T_{x} M_{1}$, in this order, form a positively oriented basis of $T_{x} M$. One can prove that this pointwise choice induces a (global) orientation on $M_{1}$ (see, e.g., [9, pages 100-101] for the details).

Definition 2.4. The submanifold $M_{1}$, oriented as above, is called the oriented $g$-preimage of $F_{1}$.

Let now $f: M \rightarrow F$ be continuous and let $y \in F$ be such that $f^{-1}(y)$ is compact. Consider a $C^{1} \operatorname{map} g: M \rightarrow F$ and a subspace $F_{1}$ of $F$ such that 
(a) $F_{1}$ contains $y$ and $(f-g)(M)$,

(b) $g$ is transverse to $F_{1}$.

Now observe that assumption (a) implies that the compact set $f^{-1}(y)$ coincides with $f_{1}^{-1}(y)$, where $f_{1}$ stands for the restriction $\left.f\right|_{M_{1}}: M_{1} \rightarrow F_{1}$. Therefore, the Brouwer degree of the triple $\left(f_{1}, M_{1}, y\right)$ is well defined.

We can now state the following reduction property of the degree. The proof of this result can be obtained following the outline of the analogous result given for maps between Euclidean spaces, where the rôle of $g$ is played by the identity of $\mathbb{R}^{n}$ (see, e.g., [13, Lemma 4.2.3]).

Proposition 2.5 (reduction). Let $M$ be an oriented manifold and $F$ an oriented vector space of the same finite dimension as $M$. Let $f: M \rightarrow F$ be continuous and $y \in F$ such that $f^{-1}(y)$ is compact. Consider an oriented subspace $F_{1}$ of $F$ and a $C^{1}$ map $g: M \rightarrow F$ such that

(1) $F_{1}$ contains $y$ and $(f-g)(M)$,

(2) $g$ is transverse to $F_{1}$.

Let $M_{1}$ denote the oriented $g$-preimage of $F_{1}$. Then

$$
\operatorname{deg}(f, M, y)=\operatorname{deg}\left(f_{1}, M_{1}, y\right)
$$

where $f_{1}$ is the restriction of $f$ to $M_{1}$ as domain and to $F_{1}$ as codomain.

\section{Orientability for Fredholm maps}

In $[1,2]$ we introduced a notion of orientability for (nonlinear) Fredholm maps of index zero between Banach manifolds. This section deals with a summary of this notion in the particular context of Fredholm maps between Banach spaces. For the details we refer to $[1,2]$.

3.1. Orientability for Fredholm linear operators. The starting point for the definition of our notion of orientability for Fredholm maps is a preliminary concept of orientation for Fredholm linear operators of index zero between real vector spaces (at this level no topological structure is needed). Given two real vector spaces $E$ and $F$, a linear operator $L: E \rightarrow F$ is said to be (algebraic) Fredholm if $\operatorname{Ker} L$ and $\operatorname{coKer} L=F / \operatorname{Im} L$ are finitedimensional. The index of $L$ is

$$
\text { ind } L=\operatorname{dim} \operatorname{Ker} L-\operatorname{dim} \operatorname{coKer} L \text {. }
$$

Of course, if $E$ and $F$ have finite dimension, then $\operatorname{ind} L=\operatorname{dim} E-\operatorname{dim} F$.

A linear operator $A: E \rightarrow F$ is a corrector of $L$ provided its image has finite dimension and $L+A$ is an isomorphism. We denote by $\mathscr{C}(L)$ the (nonempty) set of correctors of $L$. In $\mathscr{C}(L)$ an equivalence relation can be defined as follows. Given $A, B \in \mathscr{C}(L)$, consider the following automorphism of $E$ :

$$
T=(L+B)^{-1}(L+A)=I-(L+B)^{-1}(B-A) .
$$


Clearly, $K=(L+B)^{-1}(B-A)$ has finite-dimensional image. Hence, given any finitedimensional subspace $E_{0}$ of $E$ containing the image of $K$, the restriction of $T$ to $E_{0}$ is an automorphism of $E_{0}$. Therefore, its determinant is well defined and nonzero. It is easy to check that such a value does not depend on $E_{0}$ (see [1]). Thus, the number

$$
\operatorname{det}\left((L+B)^{-1}(L+A)\right)
$$

is well defined as the determinant of the restriction of $(L+B)^{-1}(L+A)$ to any finitedimensional subspace of $E$ containing the image of $(L+B)^{-1}(B-A)$.

We say that $A$ is equivalent to $B$ or, more precisely, $A$ is $L$-equivalent to $B$, if $\operatorname{det}((L+$ $\left.B)^{-1}(L+A)\right)>0$. In $[1$, Section 2$]$ it is shown that this is actually an equivalence relation on $\mathscr{C}(L)$ with two equivalence classes. This relation allows us to introduce the following concept of orientation for a Fredholm linear operator of index zero.

Definition 3.1. Let $L$ be a Fredholm linear operator of index zero between two real vector spaces. An orientation of $L$ is the choice of one of the two equivalence classes of $\mathscr{C}(L)$. We say that $L$ is oriented when an orientation is chosen.

Given an oriented operator $L$, the elements of its orientation will be called the positive correctors of $L$.

According to Definition 3.1, an oriented operator is actually a pair $(L, \alpha)$, where $L$ is a nonoriented operator and $\alpha$ is an orientation of $L$. However, to simplify the notation, we will not use different symbols to distinguish between an oriented operator and its nonoriented part.

Definition 3.2. An oriented isomorphism $L$ is said to be naturally oriented if the trivial operator is a positive corrector, and this orientation is called the natural orientation of $L$.

If $E$ and $F$ are of the same finite dimension, an orientation of $L: E \rightarrow F$ induces a pair of orientations on $E$ and $F$, up to an inversion of both of them. Indeed, let $L$ be oriented and $A$ a positive corrector. Orient $E$ and $F$ in such a way that $L+A$ is orientation preserving. Clearly, the orientations of $E$ and $F$ are defined up to an inversion of both of them. It is easy to see that any other corrector $B$ of $L$ is positive if and only if $L+B$ is orientation preserving.

The converse of the above assertion holds, that is, two orientations of $E$ and $F$ induce an orientation on any linear operator between $E$ and $F$.

In the case when $L: E \rightarrow F$ acts between two infinite-dimensional spaces, given a finitedimensional subspace $F_{1}$ of $F$ which is transverse to $L$, an orientation of $L$ induces a pair of orientations on $E_{1}=L^{-1}\left(F_{1}\right)$ and on $F_{1}$, up to an inversion of both of them. To prove this, let $E_{0}$ be a direct complement of $E_{1}$ in $E$ and observe that $F=L\left(E_{0}\right) \oplus F_{1}$. Consider a corrector $A$ of $L$ with image contained in $F_{1}$. It follows that $\left.(L+A)\right|_{E_{1}}: E_{1} \rightarrow F_{1}$ is an isomorphism. Therefore, it is possible to orient $E_{1}$ and $F_{1}$ in such a way that $\left.(L+A)\right|_{E_{1}}$ : $E_{1} \rightarrow F_{1}$ is orientation preserving.

Assume now that $L$ is oriented and $A$ is a positive corrector. Let us check that the pair of orientations induced on $E_{1}$ and on $F_{1}$ (up to an inversion of both of them) does not depend on the choice of $A$, but just on the orientation of $L$. Let $B$ be a positive corrector of 
$L$ with image contained in $F_{1}$. From the definition of the orientation of $L$ it follows that the determinant of $\left.(L+B)^{-1}(L+A)\right|_{E_{1}}: E_{1} \rightarrow E_{1}$ is positive (i.e., $A$ and $B$ are $L$-equivalent). If we now choose two bases of $E_{1}$ and of $F_{1}$ in such a way that the determinant of $\left.(L+A)\right|_{E_{1}}$ : $E_{1} \rightarrow F_{1}$ is positive, it turns out that the determinant of $\left.(L+B)^{-1}\right|_{F_{1}}: F_{1} \rightarrow E_{1}$ is positive as well. Hence the determinant of its inverse $\left.(L+B)\right|_{E_{1}}: E_{1} \rightarrow F_{1}$ is positive.

In conclusion, given two correctors $A$ and $B$ of $L$ with images contained in $F_{1}$, if we orient $E_{1}$ and $F_{1}$ in such a way that $\left.(L+A)\right|_{E_{1}}: E_{1} \rightarrow F_{1}$ preserves the orientations, then $\left.(L+B)\right|_{E_{1}}: E_{1} \rightarrow F_{1}$ preserves the orientations if and only if $B$ is equivalent to $A$.

3.2. Orientability for Fredholm maps. We now extend the notion of orientation in the framework of Banach spaces. From now on $E$ and $F$ denote two real Banach spaces, $L(E, F)$ is the Banach space of bounded linear operators from $E$ into $F$, and $\Phi_{n}(E, F)$ is the open subset of $L(E, F)$ of the Fredholm operators of index $n$. Given $L \in \Phi_{0}(E, F)$, the symbol $\mathscr{C}(L)$ now denotes, with an abuse of notation, the set of bounded correctors of $L$, which is still nonempty.

Of course, the definition of orientation of $L \in \Phi_{0}(E, F)$ can be given as the choice of one of the two equivalence classes of bounded correctors of $L$, according to the equivalence relation previously defined.

In the context of Banach spaces, an orientation of a Fredholm linear operator of index zero induces, by a sort of stability, an orientation to any sufficiently close operator. Precisely, consider $L \in \Phi_{0}(E, F)$ and a corrector $A$ of $L$. Since the set of the isomorphisms from $E$ into $F$ is open in $L(E, F), A$ is a corrector of every $T$ in a suitable neighborhood $U$ of $L$. If, in addition, $L$ is oriented and $A$ is a positive corrector of $L$, then any $T$ in $U$ can be oriented taking $A$ as a positive corrector. This fact leads us to the following notion of orientation for a continuous map with values in $\Phi_{0}(E, F)$.

Definition 3.3. Let $X$ be a topological space and $h: X \rightarrow \Phi_{0}(E, F)$ be continuous. An orientation of $h$ is a continuous choice of an orientation $\alpha(x)$ of $h(x)$ for each $x \in X$, where "continuous" means that for any $x \in X$ there exists $A \in \alpha(x)$ which is a positive corrector of $h\left(x^{\prime}\right)$ for any $x^{\prime}$ in a neighborhood of $x$. A map is orientable when it admits an orientation and oriented when an orientation is chosen.

Remark 3.4. It is possible to prove (see [2, Proposition 3.4]) that two equivalent correctors $A$ and $B$ of a given $L \in \Phi_{0}(E, F)$ remain $T$-equivalent for any $T$ in a neighborhood of $L$. This implies that the notion of "continuous choice of an orientation" in Definition 3.3 is equivalent to the following one:

(i) for any $x \in X$ and any $A \in \alpha(x)$, there exists a neighborhood $U$ of $x$ such that $A \in$ $\alpha\left(x^{\prime}\right)$ for all $x^{\prime} \in U$.

As a straightforward consequence of Definition 3.3, if $h: X \rightarrow \Phi_{0}(E, F)$ is orientable and $g: Y \rightarrow X$ is any continuous map, then the composition $h \circ g$ is orientable as well. In particular, if $h$ is oriented, then $h \circ g$ inherits in a natural way an orientation from the orientation of $h$. This holds, for example, for the restriction of $h$ to any subset $A$ of $X$, since $\left.h\right|_{A}$ is the composition of $h$ with the inclusion $A \hookrightarrow X$. Moreover, if $H: X \times$ $[0,1] \rightarrow \Phi_{0}(E, F)$ is an oriented homotopy and $\lambda \in[0,1]$, the partial map $H_{\lambda}=H \circ i_{\lambda}$, where $i_{\lambda}(x)=(x, \lambda)$, inherits an orientation from $H$. 
The following proposition shows an important property of the notion of orientability for continuous maps in $\Phi_{0}(E, F)$, which is, roughly speaking, a sort of continuous transport of an orientation along a homotopy (see [2, Theorem 3.14]).

Proposition 3.5. Consider a homotopy $H: X \times[0,1] \rightarrow \Phi_{0}(E, F)$. If, for some $\lambda \in[0,1]$, the partial map $H_{\lambda}=H(\cdot, \lambda)$ is oriented, then there exists a unique orientation of $H$ such that the orientation of $H_{\lambda}$ is inherited from that of $H$.

Definition 3.3 and Remark 3.4 allow us to define a notion of orientability for Fredholm maps of index zero between Banach spaces. Recall that, given an open subset $\Omega$ of $E$, a map $g: \Omega \rightarrow F$ is a Fredholm map if it is $C^{1}$ and its Fréchet derivative, $g^{\prime}(x)$, is a Fredholm operator for all $x \in \Omega$. The index of $g$ at $x$ is the index of $g^{\prime}(x)$ and $g$ is said to be of index $n$ if it is of index $n$ at any point of its domain.

Definition 3.6. An orientation of a Fredholm map of index zero $g: \Omega \rightarrow F$ is an orientation of the continuous map $g^{\prime}: x \mapsto g^{\prime}(x)$, and $g$ is orientable, or oriented, if so is $g^{\prime}$ according to Definition 3.3.

The notion of orientability of Fredholm maps of index zero is mainly discussed in $[1,2]$, where the reader can find examples of orientable and nonorientable maps. It is worthwhile for the sequel to recall the following sufficient condition for the orientability of a Fredholm map (see [1]).

Proposition 3.7. A Fredholm map of index zero $g: \Omega \rightarrow F$ is orientable if $\Omega$ is simply connected.

Let us now recall a property (Theorem 3.9 below) which is the analogue for Fredholm maps of the continuous transport of an orientation along a homotopy, as seen in Proposition 3.5. We need first the following definition.

Definition 3.8. Let $H: \Omega \times[0,1] \rightarrow F$ be a $C^{1}$ homotopy. Assume that any partial map $H_{\lambda}$ is Fredholm of index zero. An orientation of $H$ is an orientation of the map

$$
\partial_{1} H: \Omega \times[0,1] \longrightarrow \Phi_{0}(E, F), \quad(x, \lambda) \longmapsto\left(H_{\lambda}\right)^{\prime}(x)
$$

and $H$ is orientable, or oriented, if so is $\partial_{1} H$ according to Definition 3.3.

From the above definition it follows immediately that if $H$ oriented, an orientation of any partial map $H_{\lambda}$ is inherited from $H$.

The proof of Theorem 3.9 below is a straightforward consequence of Proposition 3.5.

Theorem 3.9. Let $H: \Omega \times[0,1] \rightarrow F$ be $C^{1}$ and assume that any $H_{\lambda}$ is a Fredholm map of index zero. If a given $H_{\lambda}$ is orientable, then $H$ is orientable. If, in addition, $H_{\lambda}$ is oriented, there exists and is unique an orientation of $H$ such that the orientation of $H_{\lambda}$ is inherited from that of $H$.

We conclude this section with a generalization in infinite dimension of the concept of oriented preimage seen in Section 2.

Let $g: \Omega \rightarrow F$ be an oriented map and $Z$ a finite-dimensional subspace of $F$, transverse to $g$. By classical transversality results, $M=g^{-1}(Z)$ is a differentiable manifold of the same 
dimension as $Z$. In addition, $M$ is orientable (see [1, Remark 2.5 and Lemma 3.1]). Here we just need to show how an orientation at any point of $M$ is induced by the orientation of $g$ and by a chosen orientation of $Z$.

Let $Z$ be oriented. Consider $x \in M$ and a positive corrector $A$ of $g^{\prime}(x)$ with image contained in $Z$ (the existence of such a corrector is ensured by the transversality of $Z$ to $g$ ). Then, orient the tangent space $T_{x} M$ in such a way that the isomorphism

$$
\left.\left(g^{\prime}(x)+A\right)\right|_{T_{x} M}: T_{x} M \longrightarrow Z
$$

is orientation preserving. By the argument seen at the end of Section 3.1, the orientation of $T_{x} M$ does not depend on the choice of the positive corrector $A$, but just on the orientation of $Z$ and $g^{\prime}(x)$. With this orientation, we call $M$ the oriented Fredholm $g$-preimage of $Z$.

The reader can immediately notice the similarity between the above notion of oriented Fredholm preimage and that of oriented preimage given in Section 2. In both cases we assign an orientation to a finite-dimensional manifold obtained as preimage of a suitable finite-dimensional oriented vector space. However, in the notion of oriented preimage given in Section 2 the starting point is a map between two oriented finite-dimensional manifolds, while for the above notion of oriented Fredholm preimage we start from an oriented map (according to Definition 3.6).

These two definitions are formally different but strictly related, as the following lemma shows. This will be crucial for the construction of the degree of locally compact perturbations of Fredholm maps of index zero.

Lemma 3.10. Let $g: \Omega \rightarrow F$ be an oriented map and let $F_{1}$ and $F_{2}$ be two oriented finitedimensional subspaces of $F$, both transverse to $g$. Suppose that $F_{2}$ contains $F_{1}$. Let $M_{2}$ be the oriented Fredholm $g$-preimage of $F_{2}$ and put

$$
M_{1}=\left(\left.g\right|_{M_{2}}\right)^{-1}\left(F_{1}\right)=g^{-1}\left(F_{1}\right) .
$$

Then, $M_{1}$ is the oriented $\left.g\right|_{M_{2}}$-preimage of $F_{1}$ if and only if it is the oriented Fredholm $g$ preimage of $F_{1}$.

Proof. Let $x \in M_{1}$ be given and let $A: E \rightarrow F$ be a positive corrector of $g^{\prime}(x)$ having image contained in $F_{1}$. Since $M_{2}$ is the oriented Fredholm $g$-preimage of $F_{2}$, the linear operator

$$
\left.\left(g^{\prime}(x)+A\right)\right|_{T_{x} M_{2}}: T_{x} M_{2} \longrightarrow F_{2}
$$

is orientation preserving. Consider the splittings

$$
\begin{aligned}
T_{x} M_{2} & =E_{0} \oplus T_{x} M_{1}, \\
F_{2} & =F_{0} \oplus F_{1},
\end{aligned}
$$

where $E_{0}$ is any direct complement of $T_{x} M_{1}$ in $T_{x} M_{2}$ and $F_{0}=g^{\prime}(x)\left(E_{0}\right)$. By this decomposition, $\left.\left(g^{\prime}(x)+A\right)\right|_{T_{x} M_{2}}$ can be represented in a block matrix form as

$$
\left(\begin{array}{cc}
g^{\prime}(x)_{11} & 0 \\
\left(g^{\prime}(x)+A\right)_{21} & \left.\left(g^{\prime}(x)+A\right)\right|_{T_{x} M_{1}}
\end{array}\right) .
$$


Observe that $g^{\prime}(x)_{11}$ is an isomorphism. Now, orient $F_{0}$ in such a way that its positively oriented basis and a positively oriented basis of $F_{1}$, in this order, form a positively oriented basis of $F_{2}$. Then, orient $E_{0}$ in such a way that $g^{\prime}(x)_{11}$ is orientation preserving. By the notion of oriented preimage given in Section 2, $M_{1}$ is the oriented $\left.g\right|_{M_{2}}$-preimage of $F_{1}$ if and only if a positively oriented basis of $E_{0}$ and a positively oriented basis of $T_{x} M_{1}$, in this order, form a positively oriented basis of $T_{x} M_{2}$. On the other hand, $M_{1}$ is the oriented Fredholm $g$-preimage of $F_{1}$ if and only if $\left.\left(g^{\prime}(x)+A\right)\right|_{T_{x} M_{1}}: T_{x} M_{1} \rightarrow F_{1}$ is orientation preserving.

Now, by the block matrix decomposition, it is not difficult to check that, as $g^{\prime}(x)_{11}$ and $\left.\left(g^{\prime}(x)+A\right)\right|_{T_{x} M_{2}}: T_{x} M_{2} \rightarrow F_{2}$ are orientation preserving, an assigned orientation to $T_{x} M_{1}$ implies that $\left.\left(g^{\prime}(x)+A\right)\right|_{T_{x} M_{1}}: T_{x} M_{1} \rightarrow F_{1}$ is orientation preserving if and only if a positively oriented basis of $E_{0}$ and a positively oriented basis of $T_{x} M_{1}$, in this order, form a positively oriented basis of $T_{x} M_{2}$, and this completes the proof.

\section{Orientability for quasi-Fredholm maps}

In this section we introduce a concept of orientation for locally compact perturbations of Fredholm maps of index zero, in the sequel called quasi-Fredholm maps (for short, we omit the phrase "of index zero").

We recall that a map between two topological spaces is called locally compact if any point in its domain has a neighborhood whose image has compact closure. A map is compact if its image is contained in a compact set.

Definition 4.1. Let $E$ and $F$ be two real Banach spaces and $\Omega$ an open subset of $E$. Let $g: \Omega \rightarrow F$ be a Fredholm map of index zero and $k: \Omega \rightarrow F$ a locally compact map. The map $f: \Omega \rightarrow F$, defined by $f=g-k$, is called a quasi-Fredholm map and $g$ is a smoothing map of $f$.

Definition 4.2. A quasi-Fredholm map $f: \Omega \rightarrow F$ is orientable if it has an orientable smoothing map.

If $f$ is an orientable quasi-Fredholm map, any smoothing map of $f$ is orientable. Indeed, given two smoothing maps $g_{0}$ and $g_{1}$ of $f$, consider the homotopy $H: \Omega \times[0,1] \rightarrow$ $F$, defined by

$$
H(x, \lambda)=(1-\lambda) g_{0}(x)+\lambda g_{1}(x)
$$

Notice that any $H_{\lambda}$ is Fredholm of index zero, since it differs from $g_{0}$ by a $C^{1}$ locally compact map. By Theorem 3.9, if $g_{0}$ is orientable, then $g_{1}$ is orientable as well.

Let $f: \Omega \rightarrow F$ be an orientable quasi-Fredholm map. To define a notion of orientation of $f$, consider the set $\mathscr{S}(f)$ of the oriented smoothing maps of $f$. We introduce in $\mathscr{Y}(f)$ the following equivalence relation. Given $g_{0}, g_{1}$ in $\mathscr{S}(f)$, consider, as in formula (4.1), the straight-line homotopy $H$ joining $g_{0}$ and $g_{1}$. We say that $g_{0}$ is equivalent to $g_{1}$ if their orientations are inherited from the same orientation of $H$, whose existence is ensured by Theorem 3.9. It is immediate to verify that this is an equivalence relation. If the domain of $f$ is connected, any smoothing map has two orientations and, hence, $\mathscr{S}(f)$ has exactly two equivalence classes. 
Definition 4.3. Let $f: \Omega \rightarrow F$ be an orientable quasi-Fredholm map. An orientation of $f$ is the choice of an equivalence class in $\mathscr{Y}(f)$.

By the above construction, given an orientable quasi-Fredholm map $f$ and a smoothing map $g$, an orientation of $g$ determines uniquely an orientation of $f$. Therefore, in the sequel, if $f$ is oriented, we will refer to a positively oriented smoothing map of $f$ as an element in the chosen class of $\mathscr{Y}(f)$.

As for Fredholm maps of index zero, the orientation of quasi-Fredholm maps enjoys a homotopy invariance property, as shown in Theorem 4.6 below. We need first some definitions.

Definition 4.4. Let $H: \Omega \times[0,1] \rightarrow F$ be a map of the form

$$
H(x, \lambda)=G(x, \lambda)-K(x, \lambda)
$$

where $G$ is $C^{1}$, any $G_{\lambda}$ is Fredholm of index zero and $K$ is locally compact. We call $H$ a homotopy of quasi-Fredholm maps and $G$ a smoothing homotopy of $H$.

We need a concept of orientability for homotopies of quasi-Fredholm maps. The definition is analogous to that given for quasi-Fredholm maps. Let $H: \Omega \times[0,1] \rightarrow F$ be a homotopy of quasi-Fredholm maps. Let $\mathscr{S}(H)$ be the set of oriented smoothing homotopies of $H$. Assume that $\mathscr{S}(H)$ is nonempty and define on this set an equivalence relation as follows. Given $G_{0}$ and $G_{1}$ in $\mathscr{S}(H)$, consider the map

$$
\mathscr{H}: \Omega \times[0,1] \times[0,1] \longrightarrow F
$$

defined as

$$
\mathscr{H}(x, \lambda, s)=(1-s) G_{0}(x, \lambda)+s G_{1}(x, \lambda) .
$$

We say that $G_{0}$ is equivalent to $G_{1}$ if their orientations are inherited from an orientation of the map

$$
(x, \lambda, s) \longmapsto \partial_{1} \mathscr{H}(x, \lambda, s)
$$

The reader can easily verify that this is actually an equivalence relation on $\mathscr{S}(H)$.

Definition 4.5. A homotopy of quasi-Fredholm maps $H: \Omega \times[0,1] \rightarrow F$ is said to be orientable if $\mathscr{S}(H)$ is nonempty. An orientation of $H$ is the choice of an equivalence class of $\mathscr{S}(H)$.

The following homotopy invariance property of the orientation of quasi-Fredholm maps is the analogue of Theorem 3.9. The proof is a straightforward consequence of Proposition 3.5.

Theorem 4.6. Let $H: \Omega \times[0,1] \rightarrow F$ be a homotopy of quasi-Fredholm maps. If a partial map $H_{\lambda}$ is oriented, then there exists a unique orientation of $H$ such that the orientation of $H_{\lambda}$ is inherited from that of $H$.

We conclude the section by showing an example of a homotopy of quasi-Fredholm maps. 
Example 4.7. Let $\phi:[0, T] \times \mathbb{R}^{n} \times \mathbb{R}^{n} \rightarrow \mathbb{R}^{n}$ and $\psi:[0, T] \times \mathbb{R}^{n} \rightarrow \mathbb{R}^{n}$ be of class $C^{1}$ and continuous, respectively. Denote by $\mathscr{C}^{1}$ and $\mathscr{C}^{0}$ the Banach spaces $C^{1}\left([0, T], \mathbb{R}^{n}\right)$ and $C\left([0, T], \mathbb{R}^{n}\right)$, and let

$$
\begin{array}{ll}
\tilde{G}: \mathscr{C}^{1} \times \mathbb{R} \longrightarrow \mathscr{C}^{0}, & \tilde{G}(x, \lambda)(t)=x^{\prime}(t)+\lambda \phi\left(t, x(t), x^{\prime}(t)\right), \\
\tilde{K}: \mathscr{C}^{1} \times \mathbb{R} \longrightarrow \mathscr{C}^{0}, & \tilde{K}(x, \lambda)(t)=\lambda \psi(t, x(t)) .
\end{array}
$$

The map $\widetilde{G}$ is $C^{1}$ (since so is $\phi$ ) and the Fréchet derivative $\widetilde{G}_{\lambda}^{\prime}(x): \mathscr{C}^{1} \rightarrow \mathscr{C}^{0}$ of any partial map $\tilde{G}_{\lambda}$ at any $x \in C^{1}$ is given by

$$
\left(\widetilde{G}_{\lambda}^{\prime}(x) q\right)(t)=q^{\prime}(t)+\lambda \partial_{2} \phi\left(t, x(t), x^{\prime}(t)\right) q(t)+\lambda \partial_{3} \phi\left(t, x(t), x^{\prime}(t)\right) q^{\prime}(t),
$$

where $\partial_{2} \phi$ and $\partial_{3} \phi$ denote the jacobian matrices of $\phi$ with respect to the second and third variable. Formula (4.7) can be rewritten as

$$
\left(\tilde{G}_{\lambda}^{\prime}(x) q\right)(t)=\left(I+\lambda M_{x}(t)\right) q^{\prime}(t)+\lambda N_{x}(t) q(t)
$$

where $I$ is the $n \times n$ identity matrix and, given $x \in \mathscr{C}^{1}, M_{x}$ and $N_{x}$ are $n \times n$ matrices of continuous real functions defined in $[0, T]$. Clearly, if $x$ and $\lambda$ are such that

$$
\operatorname{det}\left(I+\lambda M_{x}(t)\right) \neq 0, \quad \forall t \in[0, T]
$$

then $\widetilde{G}_{\lambda}^{\prime}(x): \mathscr{C}^{1} \rightarrow \mathscr{C}^{0}$ is a first order linear differential operator and, consequently, it is onto with $n$-dimensional kernel.

Consider now the boundary operator

$$
B: \mathscr{C}^{1} \longrightarrow \mathbb{R}^{n}, \quad B(x)=x(T)-x(0) .
$$

Set $E=\operatorname{Ker} B$ and $F=\mathscr{C}^{0}$, and let $G, K: E \times \mathbb{R} \rightarrow F$ denote the restrictions of $\widetilde{G}$ and $\tilde{K}$ to the space $E \times \mathbb{R}$. Observe that, as $B$ is surjective, $E$ is a closed subspace of $\mathscr{C}^{1}$ of codimension $n$ and thus, for each $x \in E$ and $\lambda \in \mathbb{R}$ such that (4.9) is verified, $G_{\lambda}^{\prime}(x)$ is Fredholm of index zero. In fact, $G_{\lambda}^{\prime}(x)$ is the composition of the inclusion $E \hookrightarrow \mathscr{C}^{1}$ (which is Fredholm of index $-n)$ with $\widetilde{G}_{\lambda}^{\prime}(x)$.

Since the inclusion $\mathscr{C}^{1} \hookrightarrow \mathscr{C}^{0}$ is compact, the map $K$ is locally compact (it is actually completely continuous). Thus, if condition (4.9) is satisfied for any $x \in E$ and $\lambda \geq 0$, then

$$
H: E \times[0,+\infty) \longrightarrow F, \quad H(x, \lambda)=G(x, \lambda)+K(x, \lambda),
$$

is a homotopy of quasi-Fredholm maps (which is orientable since $E \times[0,+\infty$ ) is simply connected). This is the case if (and only if) for every

$$
(t, a, b) \in[0, T] \times \mathbb{R}^{n} \times \mathbb{R}^{n}
$$

the jacobian matrix $\partial_{3} \phi(t, a, b)$ has no negative eigenvalues. 


\section{Degree for quasi-Fredholm maps}

This section is devoted to the construction of a topological degree for oriented quasiFredholm maps. In the sequel $E$ and $F$ are real Banach spaces, $\Omega \subseteq E$ is open and $f: \Omega \rightarrow F$ is a quasi-Fredholm map.

Definition 5.1. Let $f: \Omega \rightarrow F$ be an oriented quasi-Fredholm map and $U$ an open subset of $\Omega$. The triple $(f, U, 0)$ is said to be admissible provided that $f^{-1}(0) \cap U$ is compact.

We define the degree as a map from the set of all admissible triples into $\mathbb{Z}$. The construction is divided in two steps. In the first one we consider triples $(f, U, 0)$ such that $f$ has a smoothing map $g$ with $(f-g)(U)$ contained in a finite-dimensional subspace of $F$. In the second step we remove this assumption, defining the degree for general admissible triples. In the case when $f$ is a locally compact vector field, choosing the identity as a smoothing map, our construction is similar to that of Leray-Schauder.

Step 1. Let $(f, U, 0)$ be an admissible triple and let $g$ be a positively oriented smoothing map of $f$ such that $(f-g)(U)$ is contained in a finite-dimensional subspace of $F$. As $f^{-1}(0)$ is compact, there exist a finite-dimensional subspace $Z$ of $F$ and an open neighborhood $W$ of $f^{-1}(0)$ in $U$, such that $g$ is transverse to $Z$ in $W$. We may assume that $Z$ contains $(f-g)(U)$. Let $M=g^{-1}(Z) \cap W$. As seen in Section 3, $M$ is an orientable $C^{1}$ manifold of the same dimension as $Z$. Then, let $Z$ be oriented and orient $M$ in such a way that it is the oriented Fredholm $\left.g\right|_{W}$-preimage of $Z$. One can easily verify that $\left(\left.f\right|_{M}\right)^{-1}(0)=f^{-1}(0) \cap U$. Thus $\left(\left.f\right|_{M}\right)^{-1}(0)$ is compact, and the Brouwer degree of the triple $\left(\left.f\right|_{M}, M, 0\right)$ turns out to be well defined.

Definition 5.2. Let $(f, U, 0)$ be an admissible triple and let $g$ be a positively oriented smoothing map of $f$ such that $(f-g)(U)$ is contained in a finite-dimensional subspace of $F$. Let $Z$ be a finite-dimensional subspace of $F$ and $W$ an open neighborhood of $f^{-1}(0)$ in $U$ such that

(1) $Z$ contains $(f-g)(U)$,

(2) $g$ is transverse to $Z$ in $W$.

Assume $Z$ oriented and let $M$ be the oriented Fredholm $\left.g\right|_{W}$-preimage of $Z$. Then, the degree of $(f, U, 0)$ is defined as

$$
\operatorname{deg}(f, U, 0)=\operatorname{deg}\left(\left.f\right|_{M}, M, 0\right)
$$

In order to prove that the above degree is well-defined, we have to check that the righthand side of (5.1) is independent of the choice of the smoothing map $g$, the open set $W$ and the subspace $Z$.

First of all we show that, given a smoothing map $g$, the right-hand side of (5.1) is independent of $W$ and $Z$. Fix a positively oriented smoothing map $g$ of $f$ such that $(f-g)(U)$ is contained in a finite-dimensional subspace of $F$. Once $Z$ is assigned, the independence of $W$ is a straightforward consequence of the excision property of the Brouwer degree.

Let now $Z_{1}$ and $Z_{2}$ be two oriented finite-dimensional subspaces of $F$, both containing $(f-g)(U)$, and let $W \subseteq U$ be an open neighborhood of $f^{-1}(0)$ in which $g$ is transverse 
to $Z_{1}$ and $Z_{2}$. Without loss of generality, assume that $Z_{2}$ contains $Z_{1}$ (otherwise, $Z_{2}$ can be replaced by $Z_{1}+Z_{2}$ ).

Let $M_{2}$ be the oriented Fredholm $\left.g\right|_{W}$-preimage of $Z_{2}$ and, by this orientation of $M_{2}$, let $M_{1}$ be the oriented $\left.g\right|_{M_{2}}$-preimage of $Z_{1}$. By the reduction property of the Brouwer degree (Proposition 2.5) one has

$$
\operatorname{deg}\left(\left.f\right|_{M_{1}}, M_{1}, 0\right)=\operatorname{deg}\left(\left.f\right|_{M_{2}}, M_{2}, 0\right) .
$$

On the basis of Lemma 3.10, $M_{1}$ is also the oriented Fredholm $\left.g\right|_{W}$-preimage of $Z_{1}$. Thus, once a smoothing map $g$ is assigned, the independence on $W$ and $Z$ is proved.

It remains to show the independence of the smoothing map $g$. For this purpose, consider two positively oriented smoothing maps $g_{0}$ and $g_{1}$ of $f$ such that $\left(f-g_{0}\right)(U)$ and $\left(f-g_{1}\right)(U)$ are contained in a finite-dimensional subspace of $F$. Consider the homotopy $G: \Omega \times[0,1] \rightarrow F$, defined by

$$
G(x, \lambda)=(1-\lambda) g_{0}(x)+\lambda g_{1}(x) .
$$

By the compactness of $f^{-1}(0) \cap U$, there exist an open subset $W$ of $U$, containing $f^{-1}(0) \cap$ $U$, and a finite-dimensional subspace $Z$ of $F$, containing $\left(f-g_{0}\right)(U)$ and $\left(f-g_{1}\right)(U)$, such that, for each $\lambda \in[0,1]$, the partial map $G_{\lambda}$ is transverse to $Z$ in $W$. Hence, $Z$ is transverse to $G$ in $W \times[0,1]$ and to the restriction of $G$ to the boundary of $W \times[0,1]$. Thus $G^{-1}(Z) \cap(W \times[0,1])$ is a $C^{1}$ manifold with boundary of dimension equal to $1+$ $\operatorname{dim} Z$.

Since $\left(f-g_{0}\right)(U)$ and $\left(f-g_{1}\right)(U)$ are contained in $Z$, we get $G_{\lambda}^{-1}(Z) \cap W=G_{s}^{-1}(Z) \cap$ $W$, for any $\lambda, s \in[0,1]$. Therefore $G^{-1}(Z) \cap(W \times[0,1])$ is actually a product manifold, denoted by $M \times[0,1]$, where $M=G_{\lambda}^{-1}(Z) \cap W$, for any $\lambda \in[0,1]$.

Let now $Z$ be oriented and, for any $\lambda \in[0,1]$, denote by $M_{\lambda}$ the manifold $M$ oriented in such a way that it becomes the oriented Fredholm $\left.G_{\lambda}\right|_{W}$-preimage of $Z$. The reader can imagine each $M_{\lambda}$ as the set of pairs $(x, \alpha(x, \lambda))$, where $x \in M$ and $\alpha(x, \lambda)$ is the orientation of $M$ at $x$ induced by $\left.G_{\lambda}\right|_{W}$ and $Z$.

We can prove that, for any $s, \lambda \in[0,1], M_{s}=M_{\lambda}$ (in other words, we can prove that the orientations of $M_{s}$ and $M_{\lambda}$ coincide). To see this, let $\lambda_{0} \in[0,1]$ and $\left(x, \alpha\left(x, \lambda_{0}\right)\right) \in$ $M_{\lambda_{0}}$ be given. Since $G$ is clearly oriented (with an orientation such that the orientations of $g_{0}$ and $g_{1}$ are inherited from that of $G$ ), a positive corrector $A$ of $G_{\lambda_{0}}^{\prime}(x)$ remains a positive corrector for $G_{\lambda}^{\prime}(x)$, with $\lambda$ in a suitable neighborhood of $\lambda_{0}$. Then, recalling the definition of oriented Fredholm preimage, $\alpha\left(x, \lambda_{0}\right)=\alpha(x, \lambda)$. By the connectedness of $[0,1]$, the claim follows. Therefore,

$$
\operatorname{deg}\left(\left.f\right|_{M_{0}}, M_{0}, 0\right)=\operatorname{deg}\left(\left.f\right|_{M_{1}}, M_{1}, 0\right),
$$

and thus we can say that $\operatorname{deg}(f, U, y)$ is indeed well-defined.

Step 2. Let us now extend the definition of degree to general admissible triples.

Definition 5.3 (general definition of degree). Let $(f, U, 0)$ be an admissible triple. Consider

(1) a positively oriented smoothing map $g$ of $f$; 
(2) an open neighborhood $V$ of $f^{-1}(0)$ such that $\bar{V} \subseteq U, g$ is proper on $\bar{V}$ and $(f-$ $g)\left.\right|_{V}$ is compact;

(3) a continuous map $\xi: \bar{V} \rightarrow F$ having bounded finite-dimensional image and such that

$$
\|g(x)-f(x)-\xi(x)\|<\rho, \quad \forall x \in \partial V
$$

where $\rho$ is the distance in $F$ between 0 and $f(\partial V)$. Then,

$$
\operatorname{deg}(f, U, 0)=\operatorname{deg}(g-\xi, V, 0) .
$$

First of all observe that the right-hand side of (5.6) is well defined since the triple $(g-\xi, V, 0)$ is admissible. Indeed, $g-\xi$ is proper on $\bar{V}$ and thus $(g-\xi)^{-1}(0)$ is a compact subset of $\bar{V}$ which is actually contained in $V$ by assumption (3).

We have to prove that $\operatorname{deg}(f, U, 0)$ is well-defined, in the sense that formula (5.6) does not depend on $g, \xi$ and $V$.

Consider two positively oriented smoothing maps $g_{0}$ and $g_{1}$. For $i=0,1$, let $V_{i}$ be an open neighborhood of $f^{-1}(0)$ such that $\bar{V}_{i} \subseteq U, g_{i}$ is proper on $\bar{V}_{i}$ and $\left.\left(f-g_{i}\right)\right|_{\bar{V}_{i}}$ is compact. Moreover, consider a continuous map $\xi_{i}: \bar{V}_{i} \rightarrow F$ with bounded finite-dimensional image and such that

$$
\left\|g_{i}(x)-f(x)-\xi_{i}(x)\right\|<\rho, \quad \forall x \in \partial V_{i},
$$

where $\rho$ is the distance in $F$ between 0 and the closed set $f\left(\left(\bar{V}_{0} \cup \bar{V}_{1}\right) \backslash\left(V_{0} \cap V_{1}\right)\right)$. For $i=0,1$, the map $f_{i}: \bar{V}_{i} \rightarrow F$, defined by

$$
f_{i}(x)=g_{i}(x)-\xi_{i}(x),
$$

is oriented having $g_{i}$ as positively oriented smoothing map. In addition, since $g_{i}$ is proper on $\bar{V}_{i}, f_{i}$ turns out to be proper as well. By (5.7), $f_{i}^{-1}(0)$ is a compact subset of $V_{0} \cap V_{1}$. In particular, $\left(f_{0}, V_{0}, 0\right)$ and $\left(f_{1}, V_{1}, 0\right)$ are admissible. We need to show that

$$
\operatorname{deg}\left(f_{0}, V_{0}, 0\right)=\operatorname{deg}\left(f_{1}, V_{1}, 0\right) .
$$

To see this, denoting $V=V_{0} \cap V_{1}$, define $H: \bar{V} \times[0,1] \rightarrow F$ by

$$
H(x, \lambda)=(1-\lambda) f_{0}(x)+\lambda f_{1}(x),
$$

and $G: \bar{V} \times[0,1] \rightarrow F$ by

$$
G(x, \lambda)=(1-\lambda) g_{0}(x)+\lambda g_{1}(x) .
$$

The map $H$ is proper, being a compact perturbation of $g_{0}$. Hence, $H^{-1}(0)$ is compact and, by (5.7), contained in $V \times[0,1]$. Thus there exist an open subset $W$ of $V \times[0,1]$ containing $H^{-1}(0)$, and a subspace $Z$ of $F$ of finite dimension, say $n$, containing $\xi_{0}(\bar{V})$ and $\xi_{1}(\bar{V})$ such that every partial map $G_{\lambda}$ is transverse to $Z$ on

$$
W_{\lambda}=\{x \in V:(x, \lambda) \in W\} .
$$


Consequently, the set $M=G^{-1}(Z) \cap W$ is an $(n+1)$-manifold with boundary $\left(M_{0} \times\right.$ $\{0\}) \cup\left(M_{1} \times\{1\}\right)$. In addition, the transversality of $G_{\lambda}$ to $Z$ implies that any section $M_{\lambda}$ is a boundaryless $n$-manifold.

Let $Z$ be oriented and orient $M$ in such a way that any $M_{\lambda}$ is the oriented Fredholm $G_{\lambda}$-preimage of $Z$. By Definition 5.2, one has

$$
\begin{aligned}
& \operatorname{deg}\left(f_{0}, V_{0}, 0\right)=\operatorname{deg}\left(\left.f_{0}\right|_{M_{0}}, M_{0}, 0\right), \\
& \operatorname{deg}\left(f_{1}, V_{1}, 0\right)=\operatorname{deg}\left(\left.f_{1}\right|_{M_{1}}, M_{1}, 0\right) .
\end{aligned}
$$

The homotopy invariance property of the Brouwer degree in the version of Lemma 2.2 implies

$$
\operatorname{deg}\left(\left.f_{0}\right|_{M_{0}}, M_{0}, 0\right)=\operatorname{deg}\left(\left.f_{1}\right|_{M_{1}}, M_{1}, 0\right)
$$

Therefore,

$$
\operatorname{deg}\left(f_{0}, V_{0}, 0\right)=\operatorname{deg}\left(f_{1}, V_{1}, 0\right)
$$

and we can conclude that $\operatorname{deg}(f, U, 0)$ is well-defined by (5.6).

Remark 5.4. Clearly, a Fredholm map of index zero is also quasi-Fredholm, and Definition 5.3 applies to an admissible triple $(f, U, 0)$ with $f$ of class $C^{1}$. In this case a definition of degree is given in [1] by a different approach. The reduction property proved in [1, Section 3 ] shows that the two degrees coincide when both are defined (i.e., in the $C^{1}$ case).

\section{Properties of the degree}

In this section we prove some classical properties of our concept of degree.

TheOREM 6.1. The following properties of the degree hold.

(1) (Normalization). Let $U$ be an open neighborhood of 0 in $E$ and let the identity I of $E$ be naturally oriented. Then

$$
\operatorname{deg}(I, U, 0)=1
$$

(2) (Additivity). Given an admissible triple $(f, U, 0)$ and two disjoint open subsets $U_{1}$, $U_{2}$ of $U$, such that $f^{-1}(0) \subseteq U_{1} \cup U_{2}$. Then $\left(f, U_{1}, 0\right)$ and $\left(f, U_{2}, 0\right)$ are admissible and

$$
\operatorname{deg}(f, U, 0)=\operatorname{deg}\left(f, U_{1}, 0\right)+\operatorname{deg}\left(f, U_{2}, 0\right) .
$$

(3) (Homotopy invariance). Let $H: U \times[0,1] \rightarrow F$ be an oriented homotopy of quasiFredholm maps. If $H^{-1}(0)$ is compact, then $\operatorname{deg}\left(H_{\lambda}, U, 0\right)$ is well defined and does not depend on $\lambda \in[0,1]$.

Proof. (1) This property follows immediately when we apply Definition 5.2 and recall the analogous normalization property of the Brouwer degree. 
(2) Consider

(i) a positively oriented smoothing map $g$ of $f$;

(ii) an open neighborhood $V$ of $f^{-1}(0)$ such that $\bar{V} \subseteq U, g$ is proper on $\bar{V}$ and $\left.(f-g)\right|_{\bar{V}}$ is compact;

(iii) a continuous map $\xi: \bar{V} \rightarrow F$ having bounded finite-dimensional image and such that

$$
\|g(x)-f(x)-\xi(x)\|<\rho, \quad \forall x \in \partial V
$$

where $\rho$ is the distance in $F$ between 0 and $f(\partial V)$.

Then, by Definition 5.3,

$$
\operatorname{deg}(f, U, 0)=\operatorname{deg}(g-\xi, V, 0) .
$$

In addition, we have

$$
\begin{aligned}
& \operatorname{deg}\left(f, U_{1}, 0\right)=\operatorname{deg}\left(g-\xi, V \cap U_{1}, 0\right), \\
& \operatorname{deg}\left(f, U_{2}, 0\right)=\operatorname{deg}\left(g-\xi, V \cap U_{2}, 0\right) .
\end{aligned}
$$

Let $Z$ be a finite-dimensional subspace of $F$ and $W$ be an open neighborhood of $f^{-1}(0)$ in $V$ such that

(a) $Z$ contains $\xi(W)$,

(b) $g$ is transverse to $Z$ in $W$.

Assume $Z$ oriented and let $M$ be the oriented Fredholm $\left.g\right|_{W}$-preimage of $Z$. It follows that

$$
\operatorname{deg}(g-\xi, W, 0)=\operatorname{deg}\left(\left.(g-\xi)\right|_{M}, M, 0\right)
$$

and, in addition,

$$
\begin{aligned}
& \operatorname{deg}\left(g-\xi, W \cap U_{1}, 0\right)=\operatorname{deg}\left(\left.(g-\xi)\right|_{M \cap U_{1}}, M \cap U_{1}, 0\right), \\
& \operatorname{deg}\left(g-\xi, W \cap U_{2}, 0\right)=\operatorname{deg}\left(\left.(g-\xi)\right|_{M \cap U_{2}}, M \cap U_{2}, 0\right) .
\end{aligned}
$$

The claim now follows from the additivity property of the Brouwer degree.

(3) Let $H=G-K$, where $G$ is $C^{1}$ and such that any partial map $G_{\lambda}$ of $G$ is Fredholm of index zero and $K$ is locally compact. Moreover, let any partial map of $G$ be a positively oriented smoothing map of $H_{\lambda}$. Since the projection $S$ in $E$ of $H^{-1}(0)$ is a compact subset of $U$, there exists an open neighborhood $V$ of $S$, with $\bar{V} \subseteq U$, such that $G$ is proper and $K$ is compact on $\bar{V} \times[0,1]$.

Let $\Xi: \bar{V} \times[0,1] \rightarrow F$ be a continuous map having bounded finite-dimensional image and such that $\|K(x, \lambda)-\Xi(x, \lambda)\|<\rho$, for each $(x, \lambda) \in \partial V \times[0,1]$, where $\rho$ is the distance in $F$ between 0 and $H(\partial V \times[0,1])$. By Definition 5.3 , we have

$$
\operatorname{deg}\left(H_{\lambda}, U, 0\right)=\operatorname{deg}\left(G_{\lambda}-\Xi_{\lambda}, V_{\lambda}, 0\right), \quad \forall \lambda \in[0,1] .
$$

By the compactness of $H^{-1}(0)$, there exist an open subset $W$ of $V \times[0,1]$ containing $H^{-1}(0)$, and a subspace $Z$ of $F$ of finite dimension, say $n$, containing $\Xi(\bar{V} \times[0,1])$ such 
that every partial map $G_{\lambda}$ is transverse to $Z$ on $W_{\lambda}$. Consequently, the set $M=G^{-1}(Z) \cap$ $W$ is an $(n+1)$-manifold with boundary $\left(M_{0} \times\{0\}\right) \cup\left(M_{1} \times\{1\}\right)$.

Let $Z$ be oriented and orient $M$ in such a way that any $M_{\lambda}$ is the oriented Fredholm $G_{\lambda}$-preimage of $Z$. By Definition 5.2, one has

$$
\begin{aligned}
& \operatorname{deg}\left(G_{0}-\Xi_{0}, W_{0}, 0\right)=\operatorname{deg}\left(G_{0}-\Xi_{0}, M_{0}, 0\right), \\
& \operatorname{deg}\left(G_{1}-\Xi_{1}, W_{1}, 0\right)=\operatorname{deg}\left(G_{1}-\Xi_{1}, M_{1}, 0\right) .
\end{aligned}
$$

The homotopy invariance property of the Brouwer degree in the version of Lemma 2.2 implies

$$
\operatorname{deg}\left(G_{0}-\Xi_{0}, M_{0}, 0\right)=\operatorname{deg}\left(G_{1}-\Xi_{1}, M_{1}, 0\right),
$$

and the proof is complete.

We observe that the degree can be immediately extended to triples of the form $(f, U, h)$, where $h$ is a locally compact map such that $(f-h, U, 0)$ is admissible according to Definition 5.1. In this case we define

$$
\operatorname{deg}(f, U, h)=\operatorname{deg}(f-h, U, 0) .
$$

In particular, if $y$ is a point of the target space $F$, we obtain

$$
\operatorname{deg}(f, U, y)=\operatorname{deg}(f-y, U, 0)
$$

provided that $f^{-1}(y) \cap U$ is compact.

We conclude the section by providing an application of the degree to the following boundary value problem depending on a parameter $\lambda \geq 0$ :

$$
\begin{gathered}
x^{\prime}(t)+\lambda \phi\left(t, x(t), x^{\prime}(t)\right)+\lambda \psi(t, x(t))=0, \\
x(0)=x(T),
\end{gathered}
$$

where $\phi: \mathbb{R} \times \mathbb{R}^{n} \times \mathbb{R}^{n} \rightarrow \mathbb{R}^{n}$ and $\psi: \mathbb{R} \times \mathbb{R}^{n} \rightarrow \mathbb{R}^{n}$ are as in Example 4.7. Notice that if $\phi$ and $\psi$ are $T$-periodic with respect to the first variable, any solution of (6.13) is a $T$ periodic $\left(\mathbb{R}^{n}\right.$-valued) function.

For technical reasons define

$$
\begin{array}{ll}
L: E \longrightarrow F, & L x(t)=x^{\prime}(t), \\
h: E \longrightarrow F, & h(x)(t)=\phi\left(t, x(t), x^{\prime}(t)\right), \\
k: E \longrightarrow F, & k(x)(t)=\psi(t, x(t)),
\end{array}
$$

where the Banach spaces $E$ and $F$ are as in Example 4.7. Thus, problem (6.13) is equivalent to the semilinear operator equation

$$
L x+\lambda(h(x)+k(x))=0
$$


in $E \times[0,+\infty)$. We assume that, for any $(t, a, b) \in[0, T] \times \mathbb{R}^{n} \times \mathbb{R}^{n}$, the jacobian matrix $\partial_{3} \phi(t, a, b)$ has no negative eigenvalues; so that, as in Example 4.7,

$$
H(x, \lambda)=L x+\lambda(h(x)+k(x))
$$

is an orientable homotopy of quasi-Fredholm maps (with $G:(x, \lambda) \mapsto L x+\lambda h(x)$ of class $C^{1}$ and $K:(x, \lambda) \mapsto \lambda k(x)$ locally compact $)$.

By a solution of $(6.15)$ we mean a pair $(x, \lambda) \in H^{-1}(0)$ and we regard the distinguished subset $\operatorname{Ker} L \times\{0\}$ of $H^{-1}(0)$ as the set of trivial solutions of (6.15). An interesting problem related to (6.15) is that of the existence of a atypical bifurcation point; that is, a point $p$ in $\operatorname{Ker} L$ such that $(p, 0)$ lies in the closure of the set of nontrivial solutions (see [17]). In a recent paper [3] a global bifurcation result for (6.15) is obtained in the absence of the locally compact map $k$ (a map associated to the continuous function $\psi$, which is assumed identically zero in [3]). Theorem 6.2 below extends that result (by considering $\psi)$. The proof is omitted since can be carried out as in [3] just by replacing the degree for Fredholm maps (introduced by the authors in $[1,2]$ ) with the degree defined in this paper. To avoid cumbersome notation, any point $p \in \mathbb{R}^{n}$ is identified with the constant function $t \mapsto p$, so that $\mathbb{R}^{n}$ can be regarded as the set of trivial solutions of (6.15).

Theorem 6.2. Let $v: \mathbb{R}^{n} \rightarrow \mathbb{R}^{n}$ be the vector field defined by

$$
v(p)=\frac{1}{T} \int_{0}^{T}(\phi(t, p, 0)+\psi(t, p)) d t
$$

Let $U$ be an open subset of $E \times[0,+\infty)$ and let $U_{0}=\left\{p \in \mathbb{R}^{n}:(p, 0) \in U\right\}$. Assume that the Brouwer degree $\operatorname{deg}\left(v, U_{0}, 0\right)$ is defined and different from zero. Then $U$ contains a connected set of nontrivial solutions of (6.15) whose closure in $U$ is not compact and intersects $\operatorname{Ker} L \times$ $\{0\} \cong \mathbb{R}^{n}$ in the compact set $v^{-1}(0) \cap U_{0}$. In particular $U_{0}$ contains at least one atypical bifurcation point.

\section{Comparison with the Leray-Schauder degree}

The purpose of this section is to show that our degree extends the Leray-Schauder degree [12], and to clarify in what sense this extension must be interpreted.

Given a bounded open subset $U$ of a Banach space $E$ and a compact vector field $I-k$ : $\bar{U} \rightarrow E$, we recall that the Leray-Schauder degree is defined for the triple $(I-k, U, 0)$ if $x \neq k(x)$ for any $x \in \partial U$. We will call such a triple LS-admissible. Consider a continuous $\operatorname{map} \xi: \bar{U} \rightarrow E$ with finite-dimensional image and such that

$$
\|k(x)-\xi(x)\|<\rho, \quad \forall x \in \partial U
$$

where $\rho$ is the distance between 0 and $(I-k)(\partial U)$. Let $Z$ be a finite-dimensional subspace of $E$, containing the image of $\xi$. The Leray-Schauder degree of $(I-k, U, 0)$ is defined as

$$
\operatorname{deg}_{\mathrm{LS}}(I-k, U, 0)=\operatorname{deg}\left(\left.(I-\xi)\right|_{U \cap Z}, U \cap Z, 0\right),
$$

where the right-hand side is the Brouwer degree of the triple $\left(\left.(I-\xi)\right|_{U \cap Z}, U \cap Z, 0\right)$. 
The triple $(I-k, U, 0)$ is clearly admissible for our degree provided that $I-k$ is oriented (Definition 4.3). If we assign the natural orientation to $I$, it follows immediately that

$$
\operatorname{deg}(I-k, U, 0)=\operatorname{deg}_{\mathrm{LS}}(I-k, U, 0)
$$

where the left-hand side of the above equality is the degree defined in this paper. In other words, the Leray-Schauder degree of a locally compact vector field coincides with our degree if we orient $I-k$ choosing the natural orientation of the identity.

Therefore, our extension must be interpreted in a broad sense and has the following meaning: there exists a natural identification, say $i$, of the family LS of the LS-admissible triples with a subfamily of our admissible triples such that $\operatorname{deg}_{\mathrm{IS}}=\operatorname{deg} \circ i$. Namely, $i$ is the function that to any $(I-k, U, 0) \in \mathrm{LS}$ assigns the "same" triple, where the vector field $I-k$ receives the orientation induced by the natural orientation of the identity. In fact, strictly speaking, the second triple is not the same as $(I-k, U, 0) \in \mathrm{LS}$, since an oriented quasi-Fredholm map $f$ should be considered as a pair $(\tilde{f}, \alpha)$, where $\alpha$ is the orientation of $f$ and $\tilde{f}$ is its nonoriented part. However, to simplify the notation, we do not use different symbols to distinguish between an oriented quasi-Fredholm map and its nonoriented part.

As it is known, an extension of the Leray-Schauder degree to a family containing all the triples $(L, U, 0)$, where $L$ is an invertible bounded linear operator, does not exist, if the classical homotopy invariance property is required. To see this, let $E$ be a real Banach space having the set $G L(E)$ of the automorphisms connected. (An interesting result of Kuiper [11] shows that the set $G L\left(\ell^{2}\right)$ is contractible; see also [15].) In addition, consider a pair of LS-admissible triples $\left(I-K_{0}, U, 0\right)$ and $\left(I-K_{1}, U, 0\right)$ with $K_{0}$ and $K_{1}$ linear, and such that

$$
\operatorname{deg}_{\mathrm{LS}}\left(I-K_{0}, U, 0\right)=1, \quad \operatorname{deg}_{\mathrm{LS}}\left(I-K_{1}, U, 0\right)=-1 .
$$

Since there exists a continuous path in $G L(E)$ joining $I-K_{0}$ and $I-K_{1}$, the classical homotopy invariance property cannot hold.

The reader should notice that the above argument is not in contradiction with our type of extension of the Leray-Schauder degree, since our homotopy invariance property concerns just the family of the oriented homotopies. In other words, recalling the above natural identification $i$, there is no oriented homotopy $H: U \times[0,1] \rightarrow E$ joining $i(I-$ $\left.K_{0}, U, 0\right)$ to $i\left(I-K_{1}, U, 0\right)$ and such that $H^{-1}(0)$ is compact.

\section{References}

[1] P. Benevieri and M. Furi, A simple notion of orientability for Fredholm maps of index zero between Banach manifolds and degree theory, Annales des Sciences Mathématiques du Québec 22 (1998), no. 2, 131-148, dedicated to the memory of Gilles Fournier (Sherbrooke, PQ, 1997).

[2] __ On the concept of orientability for Fredholm maps between real Banach manifolds, Topological Methods in Nonlinear Analysis 16 (2000), no. 2, 279-306.

[3] P. Benevieri, M. Furi, M. Martelli, and M. P. Pera, Atypical bifurcation without compactness, Zeitschrift für Analysis und ihre Anwendungen 24 (2005), no. 1, 137-147.

[4] K. Deimling, Nonlinear Functional Analysis, Springer, Berlin, 1985. 
[5] K. D. Elworthy and A. J. Tromba, Degree theory on Banach manifolds, Nonlinear Functional Analysis (Proceedings of Sympos. Pure Math., Vol. XVIII, Part 1, Chicago, Ill., 1968) (F. E. Browder, ed.), American Mathematical Society, Rhode Island, 1970, pp. 86-94.

[6] __ Differential structures and Fredholm maps on Banach manifolds, Global Analysis (Proceedings of Sympos. Pure Math., Vol. XV, Berkeley, Calif., 1968) (S.-S. Chern and S. Smale, eds.), American Mathematical Society, Rhode Island, 1970, pp. 45-94.

[7] P. M. Fitzpatrick, J. Pejsachowicz, and P. J. Rabier, The degree of proper $C^{2}$ Fredholm mappings. I, Journal für die Reine und Angewandte Mathematik 427 (1992), 1-33.

[8] P. M. Fitzpatrick, J. Pejsachowicz, and P. Rabier, Orientability of Fredholm families and topological degree for orientable nonlinear Fredholm mappings, Journal of Functional Analysis 124 (1994), no. $1,1-39$.

[9] V. Guillemin and A. Pollack, Differential Topology, Prentice-Hall, New Jersey, 1974.

[10] M. W. Hirsch, Differential Topology, Springer, New York, 1976.

[11] N. H. Kuiper, The homotopy type of the unitary group of Hilbert space, Topology 3 (1965), 19-30.

[12] J. Leray and J. Schauder, Topologie et équations fonctionnelles, Annales Scientifiques de l'cole Normale Suprieure 51 (1934), 45-78 (French).

[13] N. G. Lloyd, Degree Theory, Cambridge University Press, Cambridge, 1978.

[14] J. W. Milnor, Topology from the Differentiable Viewpoint, The University Press of Virginia, Virginia, 1965.

[15] B. S. Mitjagin, The homotopy structure of a linear group of a Banach space, Uspekhi Matematicheskikh Nauk 25 (1970), no. 5 (155), 63-106.

[16] L. Nirenberg, Topics in Nonlinear Functional Analysis, Courant Institute of Mathematical Sciences, New York University, New York, 1974.

[17] G. Prodi and A. Ambrosetti, Analisi non lineare. I quaderno, Scuola Normale Superiore Pisa, Pisa, 1973.

[18] V. G. Zvyagin and N. M. Ratiner, Oriented degree of Fredholm maps of nonnegative index and its application to global bifurcation of solutions, Global Analysis-Studies and Applications, V (Y. G. Borisovich and Y. E. Gliklikh, eds.), Lecture Notes in Math., vol. 1520, Springer, Berlin, 1992, pp. 111-137.

Pierluigi Benevieri: Dipartimento di Matematica Applicata “G. Sansone,” Via S. Marta 3, 50139 Firenze, Italy

E-mail address: pierluigi.benevieri@unifi.it

Massimo Furi: Dipartimento di Matematica Applicata “G. Sansone,” Via S. Marta 3,

50139 Firenze, Italy

E-mail address: massimo.furi@unifi.it 


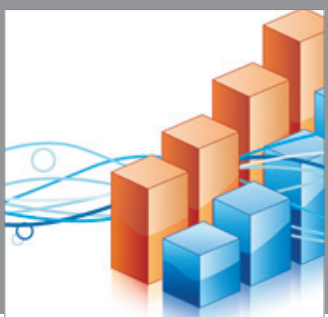

Advances in

Operations Research

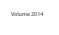

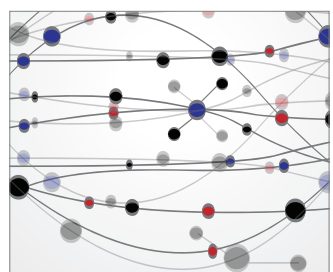

\section{The Scientific} World Journal
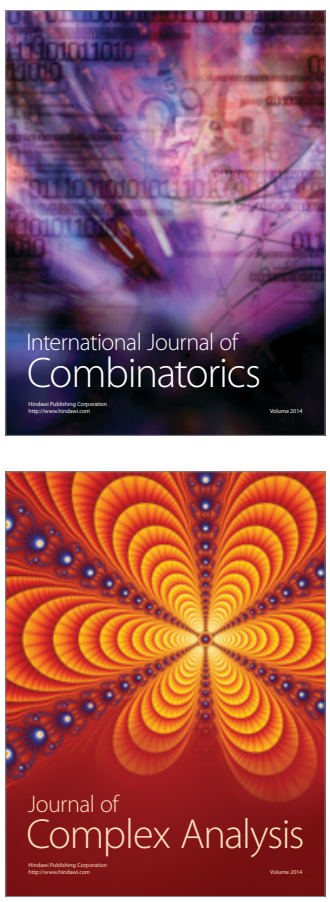

International Journal of

Mathematics and

Mathematical

Sciences
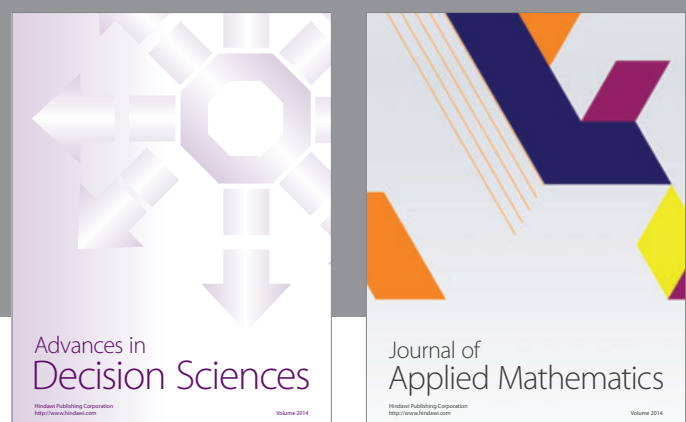

Journal of

Applied Mathematics
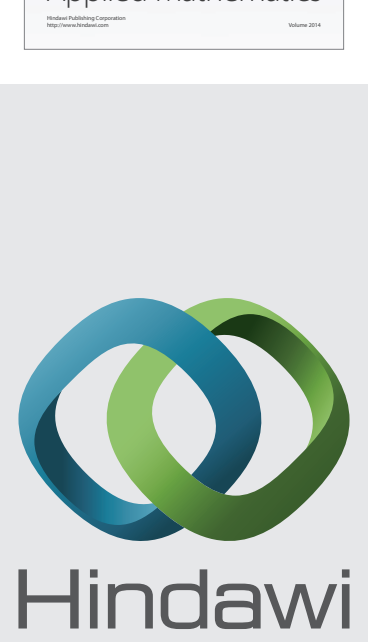

Submit your manuscripts at http://www.hindawi.com
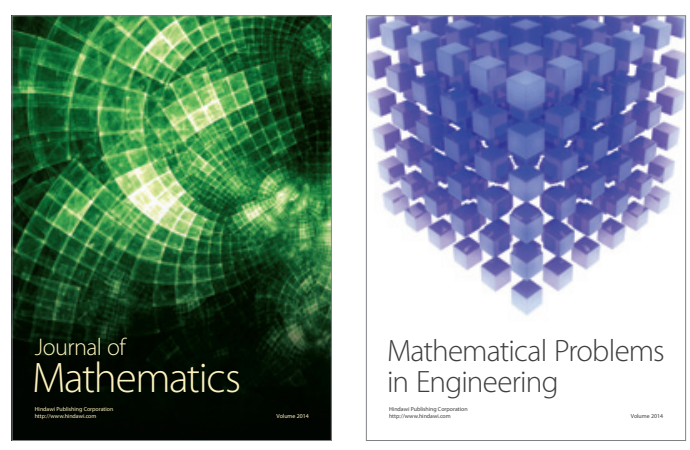

Mathematical Problems in Engineering
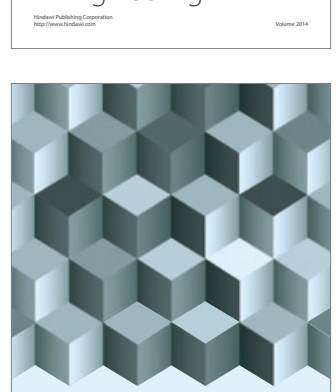

Journal of

Function Spaces
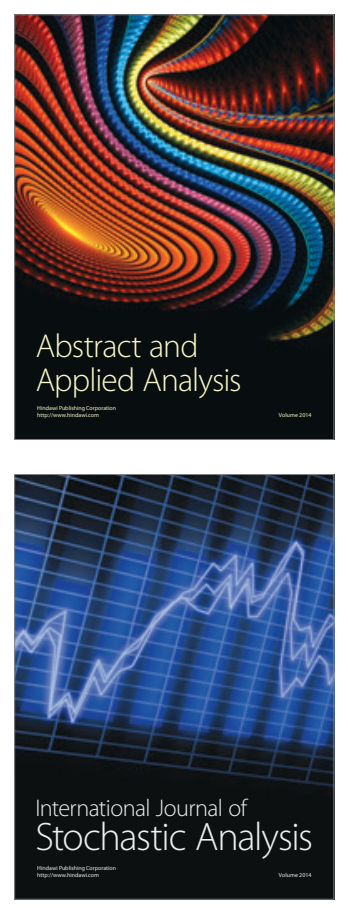

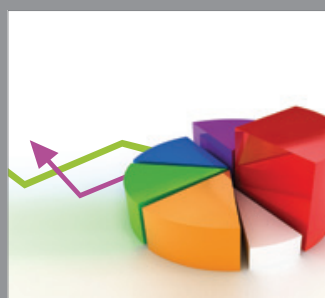

ournal of

Probability and Statistics

Promensencen
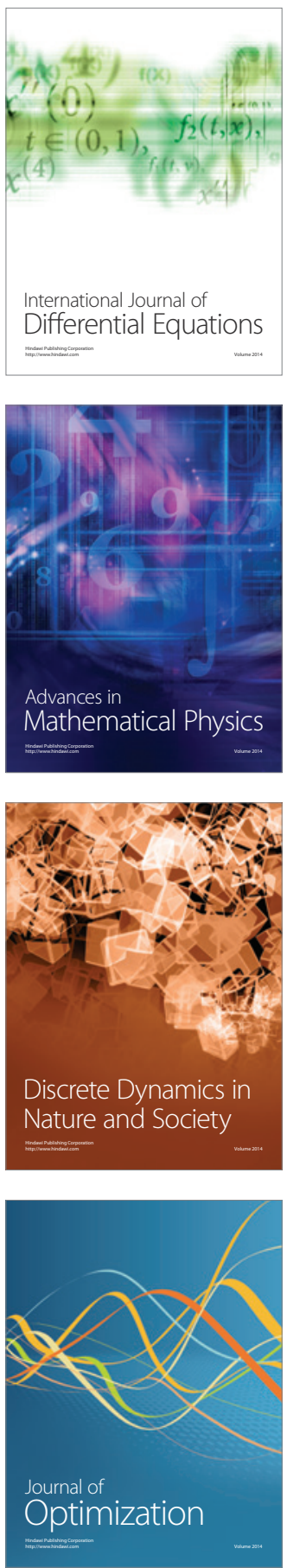